\title{
EDITORIAL
}

\section{Contours of Medical Professionalism}

\author{
Subhash Chandra Parija ${ }^{1}$, Balachandra V Adkoli
}

\begin{abstract}
One of the widely debated issues in current times is medical professionalism. While medical professionals have proved their mettle as teachers, researchers, or patient care providers, the public perception is different and plagued by mistrust. We wish to present a series of articles to paint a neutral and holistic picture of the problem. In the present article, we explore the meaning of medical professionalism, which is still an enigma for many. Next, we identify the various factors that have led to the perceived "lack of professionalism" in various domains of functioning of a medical faculty. The factors contributing to this perception are quite complex, interrelated , and often rooted outside the ambit of medical education. Nevertheless, they call for a multipronged strategy and collective participation of all stakeholders, the faculty, students, medical establishments, regulators, and civil society for upholding the image of this noble profession.
\end{abstract}

SBV Journal of Basic, Clinical and Applied Health Science (2019): 10.5005/jp-journals-10082-02231

\section{InTRODUCTION}

Professionalism in general refers to the best practices and values followed by the professionals in dealing with their clients. Every profession offers certain services to the society and, in return, gets paid for its services, in commensurate with the quality and the quantity. For defining the modalities of service, the profession lays down certain norms or code of conduct, which is nothing but professionalism. In a competitive world, where the consumer is the king, the professionals are expected to achieve not only customer satisfaction but also customer delight.

Medical profession is somewhat unique. It deals with the life and death of human beings. Historically, it is governed by certain basic code of conduct that is called medical ethics. Medical ethics forms the core of medical professionalism. The four fundamental ethical principles are patient autonomy (respect for patient), maleficence (doing no harm to the patients), beneficence (doing good), and justice (doing fairly). A doctor cannot operate on a patient without obtaining his/her informed consent. While treating, he/she should not cause any harm. The doctor should also offer a treatment that benefits the patient. At the same time, full justice should be given in deciding the priority of treatment.

While the technical knowledge and job skills constitute one dimension, the way in which they are delivered in an ethical manner forms the second dimension. Utilizing the technical expertise, and adhering to the medical ethics, a medical professional is expected to provide value-added service to the patients, which constitutes medical professionalism.

A distinction also needs to be made among the terms professionalism, morality, and law, which are intertwined with each other. While the concept of morality is a matter of individual choice, the concept of ethics governs the whole profession. Beyond morality and professional boundaries, we have laws made under legislation to safeguard the interest of all sections of the society. When professionalism is flouted, the profession has to take a call. If that doesn't happen, the law deals with the lapse as a punishable offence.

\section{Gray Area of Medical Professionalism}

When we talk about medical professionalism, what comes to our mind is a combination of competency and commitment.
${ }^{1}$ Sri Balaji Vidyapeeth (Deemed to be University), Puducherry, India

${ }^{2}$ Centre for Health Professions Education, Sri Balaji Vidyapeeth (Deemed to be University), Puducherry, India

Corresponding Author: Balachandra V Adkoli, Centre for Health Professions Education, Sri Balaji Vidyapeeth (Deemed to be University), Puducherry, India, Phone: +91 9873029295, e-mail: balachandraa@ mgmcri.ac.in

How to cite this article: Parija SC, Adkoli BV. Contours of Medical Professionalism. J Basic Clin Appl Health Sci 2019;2(4):125-127.

Source of support: Nil

Conflict of interest: The authors are on the editorial board of the SBV JBCAHS

Competency denotes the technical side and the commitment denotes the humane side of medicine. Professionalism denotes commitment to carry out professional responsibilities, adherence to ethical principles, and sensitivity to diverse population. ${ }^{1}$ It marks a shift in the emphasis from biomedical and technical aspects to the humanistic aspects of medicine.

Surprisingly, there is no universally agreed definition of professionalism. In fact, many different cultures have different shades of interpretation when they describe professional attributes. For example, eye contact is a desirable skill of a doctor in India, whereas it is not acceptable in the Middle East. However, it is possible to crystallize some commonly agreed attributes across the board. Some of the most commonly cited attributes are altruism (absence of self-interest), respect for others, humanistic qualities, empathy, duty, honesty, integrity, ethical behavior, accountability (to the profession and society), excellence (commitment to lifelong learning), communication, and collaboration. ${ }^{1,2}$ These professional attributes should become part and parcel of the various roles performed by a medical professional.

A medical professional, in general, has four domains of functioning, viz., (a) patient care provider (clinician), (b) teacher, (c) researcher, and (d) administrator. We need to examine the attributes under each of these domains that are responsible for helping or harming professional behavior. Some examples of these attributes are listed in the table (Table 1). 
Table 1: List of attributes under four domains of functioning of a medical professional

Domain-teaching/training
Fairness and transparency in setting selection/admission
procedures
procedures

Maintaining dress code decorum, punctuality, and discipline

Ensuring fair and judicious teaching and assessment modalities without any bias or favoritism, any kind of discrimination, atrocity, cynicism, professional rivalry, chauvinism

Motivating, guiding, coaching, and mentoring students by setting role models and exemplary behavior

Demonstrating proactive measures to curb disruptive student behavior (e.g., cheating, bullying/ragging, substance abuse, misuse of social media, sending hate messages, disrespect to others, ego, and arrogance)

Domain-research

Taking informed consent in all matters pertaining to the research

Demonstrate special care to be taken while researching on vulnerable subjects

Follow all ethical norms in the conduct of research

Follow publication ethics, obtaining ethical approval by the committees, collect and analyze data truthfully, avoiding falsification and fabrication of data, reporting negative findings, sticking on to authorship norms, avoiding publishing in predatory journal, plagiarism, nexus with pharma industry in funding for research

Align one's research focus on the needs of the community and society with special concern for benefiting the poor and the weaker section of society

\section{Domain-clinical (patient care)}

Demonstrating caring and compassionate attitude toward patient and role modeling exemplary behavior in teaching and clinical practice

Always put the "patients first" (taking informed consent, maintaining privacy and safety of patients)

Demonstrating exemplary behavior (restraining from any kind of misbehavior with patients, sexual advance, disclosing patient information, misuse of social media, obscene behavior)

Practicing cost-effective and quality diagnosis, treatment and care, be sensitive to socioeconomic and cultural preferences of patients in all communication

Abide by all the protocols, SOPs, best practices, regulations, and laws of the land for the benefit of patients. Raise voice against any kind of unethical behavior put up by anyone in the organization subject to approved procedure

Domain-administration

Demonstrate all the attributes of a good governance, laying down sound and transparent policies, supporting implementation by providing infrastructure and resources, sensitizing and training all concerned departments/ individuals, monitoring progress, and recognizing the contribution of everyone in the organization

Demonstrate fairness and reasonableness in charging fees for patient care or education balancing the actual costs (without compromising the quality) and the affordability of the clients

Follow the regulatory norms, in respect of governance procedures with focus on developing institutional best practices and ensuring continuous renewal of quality assurance procedures in response to the feedback from stakeholders

Develop and implement a fair policy of staff development, appraisal, career enhancement, and welfare measures for all employees

\section{Professionalism in Teaching and Training}

The issue of professionalism is rooted in quality of students who are admitted by the medical colleges and their aptitude and level of motivation. In the absence of a proper method to recruit students with high aptitude for medicine, the medical colleges are compelled to use MCQ-based tests such as NEET to select medical students on a large scale. Even assuming that the entrants are good, there is neither a standard curriculum nor the teachers who are equipped to teach professionalism. This issue is also vitiated by the fact that professionalism needs to be taught in a different way than conventional skills. The influence of role models, through what is known as "hidden curriculum" becomes very important here. ${ }^{3}$ The new Medical Education Commission has recently come out with proposals to introduce radical reforms in undergraduate medical education. One of the major planks is to start teaching of attitudes, ethics, and communication as a comprehensive longitudinal curriculum thread called AETCOM, which we will discuss later during this series. ${ }^{4}$

\section{Professionalism in Clinical Service}

Clinical services lie at the heart of medicine. Though the country has made substantial progress in health care, we cannot match the needs of our growing population in terms of providing affordable treatment to the masses. Both government and private managements are putting their best to meet the growing demands of increasing number of health-care receivers across the country. However, the spiraling cost of health-care establishments including infrastructure, maintenance and workforce, and the demand for introducing sophisticated technologies like imaging, transplants, robotics, and high-end simulations have put premium on cost of training and ethical practice. 
No doubt, our regulators have done commendable job in developing exhaustive regulations to deal with the malpractices. The Medical Council of India has framed extensive set of regulations called "Code of Ethics Regulations (2002)." ${ }^{5}$ However, what is lacking is the "will to take stringent action: against the culprits. Most regulations require concrete evidences for the lapses, which results in inordinate delay in conducting their proceedings. Of course, tribunals and consumer courts have become very active. It is therefore necessary to examine whether the punishments need to be replaced with reformatory approach that may really work.

\section{Professionalism Issues in Research Domain}

The professionalism is equally needed in research. Research is often considered as the sole criteria for the recruitment or promotion. This is largely in view of the public perception that research contributions alone can be measured objectively and fairly. While the veracity of such a claim may be debated separately, what is disturbing is the impact of this policy on the practice by the professionals. We have exhaustive guidelines laid down by Indian Council of Medical Research (National Ethical Guidelines for Biomedical and Health Research Involving Human Participants, 2017). ${ }^{6}$ It is time to work toward the modalities of implementation including sensitization, training, and monitoring activities to see that research is done in a professional manner.

\section{Professionalism in Administration And Governance}

Professionalism is indeed synonymous to good governance. Good governance is associated with good quality service delivered at an affordable cost. Unfortunately, the regulators in the past were stringent on criteria related to infrastructure, equipment, resources, and staff in place. Satisfying these requirements meant a huge investment on part of the establishments. They are forced to charge high fees in order to recover the investment made. This policy has hampered in two ways. It has discouraged launching of curricular innovations that could have cut down the cost. It has also constrained the aspirants to medicine who cannot afford to pay high fees.

Quality assurance and accreditation are the drivers for developing professionalism. The National Assessment and Accreditation Council (NAAC) has come out with a comprehensive manual for the health sciences universities. Though NAAC manual emphasizes the development of values as a guiding principle, the criteria of evaluation are exacting and boil down to a huge documentation of "evidences," which may sideline the true professional outcomes. ${ }^{7}$ Time alone will throw more light on its validity and authenticity in harnessing professionalism among students, staff, and establishments.

\section{Technology, Media, and Value Systems OF THE SOCIETY}

The professionalism in all the four domains, which we discussed in this episode, is influenced by the interplay of technology, media, and value systems of the society. In fact, they can work in both the ways in promoting or impeding professionalism. The advancement in genomics, and genetic engineering combined with artificial intelligence, may result in new innovations; at the same time, new challenges such as the COVID-19 situation have drawn a lot of attention on digital technologies, online teaching, and their optimum utilization without diluting the soft skills such as team building, communication, leadership and management of time, stress, and conflicts.

\section{Professionalism is a Collective ResponsibiLity}

In essence, there is a need for the medical professionals, regulators, government, and the civil society to sit across the board and formulate sound policies. The policies should be followed by best practices. These practices will result in a new "culture of professionalism," which becomes a part and parcel of our life. Culture cannot be created by a few individuals. It cannot be created overnight. It should constantly recognize, reward, and reinforce exemplary behavior in order to send right signal to the society. It appears that we need to apply both "top-down" and "bottoms-up" approaches to build a culture of professionalism. In either case, each one has to take responsibility and "be the agent of change" as preached and practiced by Mahatma Gandhi. Because, the issue is of heart, not merely of mind. We must wait and watch.

\section{References}

1. Kirk LM. Professionalism in medicine: definitions and considerations for teaching. Proc (Bayl Univ Med Cent) 2007;20(1):13-16. DOI: 10.1080/08998280.2007.11928225.

2. Hilton S. Medical professionalism: How can we encourage it in our students? The Clin Teach 2004;1(2):69-73. DOI: 10.1111/j.1743498X.2004.00032.x.

3. Whitcomb ME. Medical professionalism: can it be taught? Acad Med 2005;80(10):883-884. DOI: 10.1097/00001888-200510000-00001.

4. Medical Council of India. Competency based undergraduate curriculum for the Indian Medical Graduate. Medical Council of India, 2018. Available at: www.mciindia.org/CMS/information-desk/ for-colleges/ug-curriculum.

5. The Medical Council of India, "Code of Ethics Regulations (2002)".

6. Indian Council of Medical Research (National Ethical Guidelines for Biomedical and Health Research Involving Human Participants, 2017.

7. National Assessment and Accreditation Council (NAAC), Bengaluru, Manual of Health Sciences for Universities Available at www.naac. gov.in/images/docs/Manuals/HSM-University-25Mar19.docx. 